STRUCTURAL BIOLOGY COMMUNICATIONS

ISSN 2053-230X

\section{High-resolution structure of the alcohol dehydrogenase domain of the bifunctional bacterial enzyme AdhE}

\author{
Liyana Azmi, ${ }^{a}$ Eilis C. Bragginton, ${ }^{\mathrm{b}}$ Ian T. Cadby, ${ }^{\mathrm{b}}$ Olwyn Byron, ${ }^{\mathrm{a}}$ Andrew J. Roe, ${ }^{\mathrm{a} *}$ \\ Andrew L. Lovering ${ }^{b}$ and Mads Gabrielsen ${ }^{c_{*}}$
}

Received 20 January 2020

Accepted 23 July 2020

Edited by N. Sträter, University of Leipzig, Germany

Keywords: alcohol dehydrogenase; AdhE; Escherichia coli.

PDB references: alcohol dehydrogenase domain of AdhE, 6sci; NAD-bound, 6scg

SASBDB reference: alcohol dehydrogenase domain of AdhE, SASDC72

Supporting information: this article has supporting information at journals.iucr.org/f

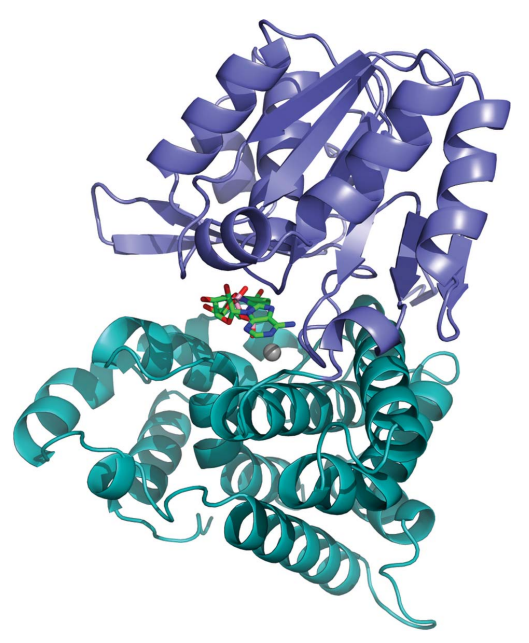

anstitute of Infection, Immunity and Inflammation, University of Glasgow, University Avenue, Glasgow G12 8QQ, United Kingdom, ${ }^{\mathbf{b}}$ School of Biosciences, University of Birmingham, Edgbaston, Birmingham B15 2TT, United Kingdom, and ${ }^{\mathrm{C} C R U K}$ Beatson Institute, Switchback Road, Glasgow G61 1BD, United Kingdom. *Correspondence e-mail: andrew.roe@glasgow.ac.uk, m.gabrielsen@beatson.gla.ac.uk

The bifunctional alcohol/aldehyde dehydrogenase (AdhE) comprises both an N-terminal aldehyde dehydrogenase (AldDH) and a C-terminal alcohol dehydrogenase $(\mathrm{ADH})$. In vivo, full-length AdhE oligomerizes into long oligomers known as spirosomes. However, structural analysis of AdhE is challenging owing to the heterogeneity of the spirosomes. Therefore, the domains of AdhE are best characterized separately. Here, the structure of ADH from the pathogenic Escherichia coli O157:H7 was determined to $1.65 \AA$ resolution. The dimeric crystal structure was confirmed in solution by smallangle X-ray scattering.

\section{Introduction}

The $96 \mathrm{kDa}$ bifunctional alcohol/aldehyde dehydrogenase (AdhE) is an intriguing protein by virtue of its structure and function. Functionally, AdhE is involved in a number of metabolic processes: mixed-acid fermentation, glycolysis, metabolism of L-threonine (Létoffé et al., 2017), purine and pyrimidine (Müller et al., 2012), and degradation of ethanolamine (Kofoid et al., 1999). AdhE is a crucial enzyme primarily in alcohol metabolism and catalyzes the conversion of the high-energy substrate acetyl-CoA to acetaldehyde and subsequently to ethanol. Structurally, full-length AdhE comprises an N-terminal aldehyde dehydrogenase (AldDH) and a C-terminal alcohol dehydrogenase (ADH) domain, and self-assembles in vivo into spiral-like structures known as spirosomes (Kawata et al., 1976; Laurenceau et al., 2015; Ueki et al., 1982). The helical structure of spirosomes is speculated to enhance the enzymatic activity of AdhE as well as to protect the protein from its toxic intermediate product, acetaldehyde (Extance et al., 2013; Kim et al., 2019). Ethanol generation by AdhE has been extensively studied in the context of biofuel production. Many fermentative and thermophilic bacteria such as Thermoanaerobacterium saccharolyticum, Clostridium thermocellum (Lo et al., 2015) and Geobacillus thermoglucosidasius (Hills, 2015) have been used as model organisms in which AdhE was genetically modified to improve the ethanol yield. Point mutations in AdhE introducing different cofactor specificities have been found to regulate ethanol generation (Tian et al., 2019; Zheng et al., 2015). Based on work by Zheng et al. (2015), an Asp-to-Gly substitution in the $\mathrm{ADH}$ domain of $G$. thermoglucosidasius (residue 494 in G. thermoglucosidasius numbering, corresponding to residue 487 in the Escherichia coli enzyme) was 
discovered to significantly increase ethanol production by changing the cofactor specificity from NADH to NADPH. The cofactor-binding region of AdhE is located within the ADH domain, suggesting the importance of the ADH domain with regard to improving ethanol production. Solving the highresolution structure of the $\mathrm{ADH}$ domain could provide important insights into how this mutation leads to the observed differential cofactor occupancies. A structure could also provide clues about the basis of spirosome assembly. Here, we describe the atomic structure of the cofactor-bound ADH domain from E. coli determined by X-ray crystallography to $1.65 \AA$ resolution. The structure of the apo form was also determined to $1.95 \AA$ resolution. Solution data obtained via small-angle $\mathrm{X}$-ray scattering agree with the crystal structures and confirm the dimeric structure of the ADH domain. Comparisons with other alcohol dehydrogenase structures revealed a loop that is involved in coordinating the domains of the bifunctional enzyme.

\section{Materials and methods}

\subsection{Macromolecule production}

The C-terminal part of the adhE gene from E. coli O157:H7 (encoding residues 451 to the C-terminus) was cloned into a p77 vector (p77-D2) which encodes a C-terminal $\mathrm{His}_{6}$ tag. The construct was transformed into E. coli BL21(DE3) cells, which were grown in lysogeny broth (LB) containing $100 \mathrm{mg} \mathrm{ml}^{-1}$ ampicillin at $37^{\circ} \mathrm{C}$ until an optical density $\left(\mathrm{OD}_{600}\right)$ of $0.6-0.8$ was reached, whereupon the cultures were induced with a $1 \mathrm{~m} M$ final concentration of isopropyl $\beta$-D-1-thiogalactopyranoside (IPTG) and left to grow at $28^{\circ} \mathrm{C}$ overnight. The cells were harvested and resuspended in $20 \mathrm{~m} M$ Tris $\mathrm{pH} 7.5$, $500 \mathrm{~m} M \mathrm{NaCl}, 5 \%(v / v)$ glycerol (buffer $A$ ) with $20 \mathrm{~m} M$ imidazole.

The cells were sonicated in the presence of $10 \mathrm{mg}$ DNAse (Sigma), $1 \mathrm{mg} \mathrm{ml}^{-1}$ EDTA-free protease inhibitors (Enzo Life Sciences) and $1 \mathrm{mg} \mathrm{ml}^{-1}$ lysozyme using $15 \mathrm{~s}$ on/off cycles, and the lysate was cleared by centrifugation and filtration. The cleared lysate was applied onto a $5 \mathrm{ml} \mathrm{Ni}{ }^{2+}$ HisTrap column (GE Healthcare) that had been pre-equilibrated in buffer $A$, and was washed in buffer $A$ plus $100 \mathrm{~m} M$ imidazole before the protein was eluted using an increasing gradient of imidazole. The purity of the protein was assessed by SDS-PAGE to be around $90-95 \%$, and the protein-containing samples were dialyzed against buffer $A$ with no imidazole but in the presence of TEV protease. Finally, the protein was loaded onto a Superdex 75 size-exclusion chromatography column (GE Healthcare) and concentrated using an Amicon Ultra 30000 molecular-weight cutoff centrifugal filter (Millipore) to $10 \mathrm{mg} \mathrm{ml}^{-1}$. The final yield of the protein was $30 \mathrm{mg}$ of purified protein from 11 of culture. Macromolecule-production information is summarized in Table 1.

\subsection{Crystallization}

Initial crystallization screens of purified ADH samples in buffer $A$ at $10 \mathrm{mg} \mathrm{ml}^{-1}$ (based on the absorbance at $280 \mathrm{~nm}$
Table 1

Macromolecule-production information.

\begin{tabular}{|c|c|}
\hline Source organism & E. coli \\
\hline DNA source & E. coli \\
\hline Forward primer & ACCATGGACATGCTGTGGCATAAGCTGCC \\
\hline Reverse primer & ACCATGGCGCGGATTTCTTC \\
\hline Cloning vector & StrataClone PCR UA \\
\hline Expression vector & p77 \\
\hline Expression host & E. coli \\
\hline $\begin{array}{l}\text { Complete amino-acid sequence } \\
\text { of the construct produced }\end{array}$ & $\begin{array}{l}\text { MDMLWHKLPKS IYFRRGSLPIALDEVITDG } \\
\text { HKRALIVTDRFLFNNGYADITSVLKAA } \\
\text { GVETEVFFEVEADPTLSIVRKGAELANS } \\
\text { FKPDVIIALGGGSPMDAAKIMWVMYEHP } \\
\text { ETHFEELARFMDIRKRIYKFPKMGVKA } \\
\text { KMIAVTTTSGTGSEVTPFAVVTDDATGQ } \\
\text { KYPLADYALTPDMAIVDANLVMDMPKSL } \\
\text { CAFGGLDAVTHAMEAYVSVLASEFSDGQ } \\
\text { ALQALKLKEYLPASYHEGSKNPVARER } \\
\text { VHSAATIAGIAFANAFLGVCHSMAHKLG } \\
\text { SQFHIPHGLANALLICNVIRYNANDNPT } \\
\text { KQTAFSQYDRPQARRYAEIADHLGLSA } \\
\text { PGDRTAKIEKLLAWLETLKALGIPKS } \\
\text { IREAGVQEADFLANVDKLSEDAFDDQCT } \\
\text { GANPRYPLISELKQILLDTYYGRDYVEG } \\
\text { ETAAKKEAAPAKAEKKAKKSAPWGAGGL } \\
\text { EVLFQGPGAAHMHHHHHHHH }\end{array}$ \\
\hline
\end{tabular}

using an extinction coefficient of $0.838 \mathrm{M}^{-1} \mathrm{~cm}^{-1}$ ) were set up against the commercial ProPlex screen (Molecular Dimensions) using the sitting-drop vapour-diffusion technique. Rodshaped crystals grew in $0.15 \mathrm{M}$ ammonium sulfate, $0.1 \mathrm{M}$ MES $\mathrm{pH} 6,15 \%(w / v)$ PEG 4000 (condition 1-22 from ProPlex). To capture crystals containing the cofactor NAD, $0.5 \mathrm{~m} M$ NAD was added to the protein before the mixture was set up against the ProPlex screen. Rod-shaped crystals initially grew in $0.2 \mathrm{M}$ lithium sulfate, $0.1 M$ MES pH 6.0, 20\%(w/v) PEG 4000 (condition 1-28 from ProPlex), which was then optimized to $0.2 M$ lithium sulfate, $0.1 M$ MES pH 5.75, $14 \%(w / v)$ PEG 4000. A small fraction of the crystal was broken off for subsequent data collection. Both crystal forms grew within $48 \mathrm{~h}$. Crystallization information is summarized in Table 2.

\subsection{Data collection and processing}

Crystals were plunge-frozen in liquid nitrogen using $40 \%(v / v)$ ethylene glycol as a cryoprotecting agent. The $P 4_{3} 2_{1} 2_{1}$ data were collected on beamline I04-1 and the $P 2_{1} 2_{1} 2_{1}$ data were collected on beamline I03 at Diamond Light Source (DLS), Didcot, UK using a PILATUS 6M detector (Dectris, Switzerland). Data were collected with $0.2^{\circ}$ oscillations for a total of 1200 images at wavelengths of $0.91741 \AA$ and $0.97957 \AA$ for the NAD-bound and apo crystals, respectively. Data were processed using MOSFLM and scaled and merged using SCALA from CCP4 (Winn et al., 2011). Data-collection and processing statistics are summarized in Table 3.

\subsection{Structure solution and refinement}

Molecular replacement used the structure of $\mathrm{ADH}$ from G. thermoglucosidasius (PDB entry 3zdr; Extance et al., 2013) as a model in Phaser (McCoy et al., 2007). Refinement was carried out in Phenix (Liebschner et al., 2019), with visual inspection and manipulations in Coot (Emsley et al., 2010). 
Table 2

Crystallization.

\begin{tabular}{|c|c|c|}
\hline & Apo & NAD-bound \\
\hline Method & Sitting-drop vapour diffusion & Sitting-drop vapour diffusion \\
\hline Plate type & CombiClover & CombiClover \\
\hline Temperature $(\mathrm{K})$ & 289 & 289 \\
\hline Protein concentration $\left(\mathrm{mg} \mathrm{ml}^{-1}\right)$ & 10 & 10 \\
\hline Buffer composition of protein solution & $20 \mathrm{~m} M$ Tris pH 7.5, $500 \mathrm{~m} M \mathrm{NaCl}, 5 \%(v / v)$ glycerol & $\begin{array}{l}20 \mathrm{~m} M \text { Tris pH } 7.5,500 \mathrm{~m} M \mathrm{NaCl}, 5 \%(v / v) \text { glycerol, } \\
0.5 \mathrm{~m} M \text { NAD }\end{array}$ \\
\hline Composition of reservoir solution & $\begin{array}{l}0.15 M \text { ammonium sulfate, } 0.1 M \text { MES pH 6.0, } \\
15 \%(w / v) \text { PEG } 4000\end{array}$ & $\begin{array}{l}0.2 M \text { lithium sulfate, } 0.1 M \text { MES pH 5.75, } \\
14 \%(w / v) \text { PEG } 4000\end{array}$ \\
\hline Volume and ratio of drop & $4 \mu \mathrm{l}, 1: 1$ ratio & $4 \mu \mathrm{l}, 1: 1$ ratio \\
\hline Volume of reservoir $(\mu \mathrm{l})^{5}$ & 140 & 140 \\
\hline
\end{tabular}

Table 3

Data collection and processing.

Values in parentheses are for the outer shell.

\begin{tabular}{|c|c|c|}
\hline & Apo & NAD-bound \\
\hline Diffraction source & I03, DLS & I04-1, DLS \\
\hline Wavelength $(\AA)$ & 0.97957 & 0.91741 \\
\hline Temperature (K) & 100 & 100 \\
\hline Detector & PILATUS 6M & PILATUS 6M \\
\hline Crystal-to-detector distance (mm) & 176 & 176 \\
\hline Rotation range per image $\left({ }^{\circ}\right)$ & 0.2 & 0.2 \\
\hline Total rotation range $\left(^{\circ}\right)$ & 240 & 240 \\
\hline Exposure time per image (s) & 0.2 & 0.2 \\
\hline Space group & $P 2_{1} 2_{1} 2_{1}$ & $P 4_{3} 2_{1} 2$ \\
\hline$a, b, c(\AA)$ & $71.03,96.73,122.89$ & $97.14,97.14,233.43$ \\
\hline$\alpha, \beta, \gamma\left({ }^{\circ}\right)$ & $90,90,90$ & $90,90,90$ \\
\hline$R_{\text {meas }}(\%)$ & $8.5(57.8)$ & $10.5(198.5)$ \\
\hline$R_{\mathrm{pim}}(\%)$ & $6.7(45.3)$ & $3.7(77.7)$ \\
\hline $\mathrm{CC}_{1 / 2}$ & $0.996(0.718)$ & $1.000(0.577)$ \\
\hline Mosaicity $\left({ }^{\circ}\right)$ & 0.1 & 0.1 \\
\hline Resolution range $(\AA)$ & $76.01-1.95$ & $50.024-1.65$ \\
\hline Total No. of reflections & 59155 & 134582 \\
\hline No. of unique reflections & 62248 & 134736 \\
\hline Completeness (\%) & 99.7 & 100 \\
\hline Multiplicity & $4.4(4.5)$ & $17.7(14.2)$ \\
\hline$\langle I / \sigma(I)\rangle$ & $10.1(2.3)$ & $19.1(1.4)$ \\
\hline $\begin{array}{l}\text { Overall } B \text { factor from Wilson } \\
\text { plot }\left(\AA^{2}\right)\end{array}$ & 34.20 & 23.37 \\
\hline
\end{tabular}

Structural superpositions were performed using LSQMAN (Kleywegt \& Jones, 1994). Refinement statistics are summarized in Table 4.

\subsection{Small-angle X-ray scattering (SAXS) data collection}

SAXS data were collected on beamline B21 at DLS with a camera length of $4.01 \mathrm{~m}$ at $12.4 \mathrm{keV}$ using a PILATUS 2M detector (Dectris, Switzerland) at a wavelength of $0.1 \mathrm{~nm}$. $50 \mu \mathrm{ADH}$ at a concentration of $10 \mathrm{mg} \mathrm{ml}^{-1}$ in buffer $A$ was loaded onto a Shodex KW-403 (molecular-mass separation range $10-700 \mathrm{kDa}$ ) size-exclusion chromatography column (Showa Denko, Japan) at $0.16 \mathrm{ml} \mathrm{min}^{-1}$ using an Agilent 1200 HPLC system. 131 successive $1.0 \mathrm{~s}$ frames of SAXS data were recorded. The data were analysed using ScAtter (http:// www.bioisis.net) as follows. The estimated radius of gyration $\left(R_{\mathrm{g}}\right)$ was plotted along with the integral of the ratio of the signal to background. A region showing a low signal-tobackground ratio (low estimated $R_{\mathrm{g}}$ ) was picked and selected as buffer, and subtracted from regions showing higher constant $R_{\mathrm{g}}$ values (indicating monodispersity) and treated as samples.
Table 4

Structure solution and refinement.

Values in parentheses are for the outer shell.

\begin{tabular}{lll}
\hline & Apo & NAD-bound \\
\hline PDB code & 6 sci & 6 scg \\
Resolution range $(\AA)$ & $76.01-1.95(2.00-1.95)$ & $50.02-1.65(1.67-1.65)$ \\
Completeness (\%) & $99.7(99.8)$ & 99.9 \\
$\sigma$ Cutoff & $F>1.36 \sigma$ & $F>1.36 \sigma$ \\
No. of reflections, working set & $59155(4370)$ & $127848(4169)$ \\
No. of reflections, test set & $3028(210)$ & $6731(216)$ \\
Final $R_{\text {cryst }}$ & $0.188(0.257)$ & $0.155(0.260)$ \\
Final $R_{\text {free }}$ & $0.207(0.252)$ & $0.181(0.274)$ \\
Cruickshank DPI & 0.132 & 0.09 \\
No. of non-H atoms & & \\
$\quad$ Protein & 6264 & 12622 \\
$\quad$ Ligand & 2 & 1004 \\
$\quad$ Water & 109 & 812 \\
R.m.s. deviations & & \\
$\quad$ Bonds $(\AA)$ & 0.011 & 0.013 \\
$\quad$ Angles $\left({ }^{\circ}\right)$ & 1.401 & 1.283 \\
Average $B$ factors $\left(\AA^{2}\right)$ & & \\
$\quad$ Protein & 34.80 & 28.13 \\
$\quad$ Ions & 21.42 & 30.00 \\
$\quad$ Ligand & $\mathrm{NA}$ & 33.43 \\
$\quad$ Water & 27.80 & 40.36 \\
Ramachandran plot & & \\
$\quad$ Most favoured $(\%)$ & 98.00 & 98.50 \\
$\quad$ Allowed $(\%)$ & 2.00 & 1.25 \\
$\quad$ Outliers $(\%)$ & 0 & 0.25 \\
\hline
\end{tabular}

Successive SAXS analysis was performed using ATSAS 2.8 (Franke et al., 2017). $R_{\mathrm{g}}$ was determined using the Guinier approximation (Guinier, 1939). The pairwise distance distribution function $p(r)$ was determined using an indirect Fourier transformation method in GNOM (Svergun, 1992). Iterative estimation of $p(r)$ allows an alternative estimation of $R_{\mathrm{g}}$ and the maximum particle dimension $D_{\text {max }}$. Rigid-body modelling of both the SAXS curve and the crystal structure of ADH were assessed using the Fast SAXS Profile Computation with Debye Formula (FoXS) server (https://modbase.compbio.ucsf.edu/ foxs/; Schneidman-Duhovny et al., 2010). All of the SAXS data analysed for ADH were deposited in SASBDB (Valentini et al., 2015) as entry SASDC72.

\section{Results and discussion}

The alcohol dehydrogenase domain of AdhE (ADH) from E. coli $\mathrm{O} 157: \mathrm{H} 7$ was crystallized in the apo form and bound to the cofactor NAD in two different crystal forms: the 
orthorhombic space group $P 22_{1} 2_{1} 2_{1}$ with unit-cell parameters $a=71.03, b=96.73, c=122.89 \AA$ and the tetragonal space group $P 4_{3} 2_{1} 2$ with unit-cell parameters $a=b=97.11$, $c=233.36 \AA$, respectively (Fig. 1). The data were processed to 1.95 and $1.65 \AA$ resolution, respectively, based on the relevant statistics (Table 3) and the structures were solved via molecular replacement. The electron density covers the majority of the residues from residue 450 (using full-length E. coli AdhE numbering) to residue 869 . There is a gap in the electron density for both structures from residues 755 to 769 , as well as missing electron density for the last 20 residues. It is assumed that these regions are particularly flexible or disordered.

The two forms superimpose well, with a root-mean-square deviation (r.m.s.d.) of $0.32 \AA$ over $404 \mathrm{C} \alpha$ atoms, and interestingly there are no obvious local differences in the binding site for NAD. The following discussion will therefore focus on the NAD-bound structure unless otherwise stated. The structure of $\mathrm{ADH}$ is similar to that of the homologous domain from G. thermoglucosidasius AdhE (gADH; Extance et al., 2013; PDB entry 3zdr), with an r.m.s.d. of $1.2 \AA$ over $373 \mathrm{C} \alpha$ atoms when superposed. The main differences between ADH from E. coli and the previous structure are around the disordered loop, which is also observed in $\mathrm{gADH}$, the region between residues 578 and 587, and an inserted proline at position 787 in the E. coli structure compared with the structure of gADH. Otherwise the folds are highly conserved.

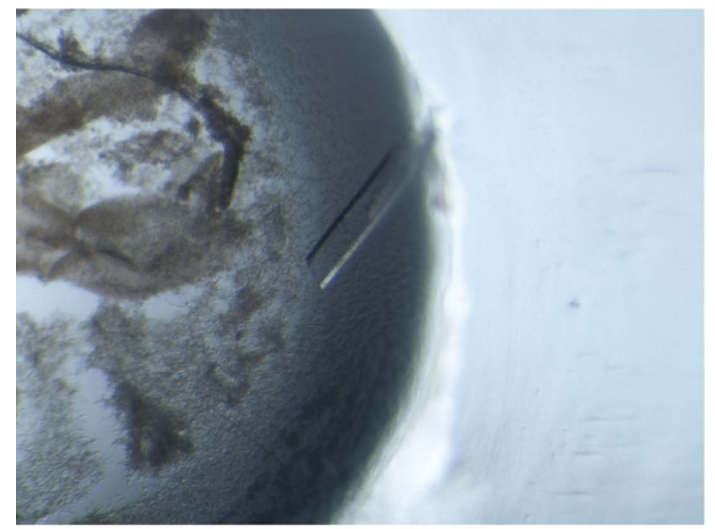

(a)

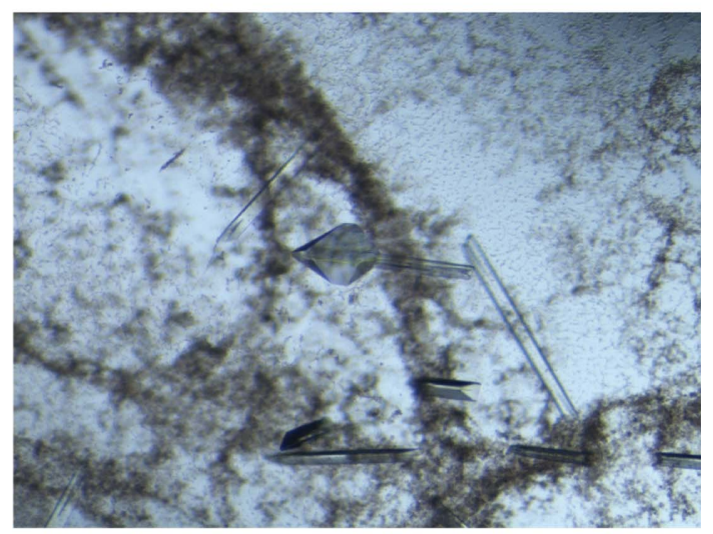

(b)

Figure 1

Crystals of ADH from E. coli. (a) Orthorhombic space group $P 22_{1} 2_{1} 2_{1},(b)$ tetragonal space group $P 4_{3} 2_{1} 2_{1}$.
Structurally, ADH comprises two subdomains: an N-terminal Rossmann-like fold (residues 450-640), in which two parallel $\beta$-sheets are sandwiched between five $\alpha$-helices, and a bundle of $11 \alpha$-helices (Fig. 2a). The surface between the two

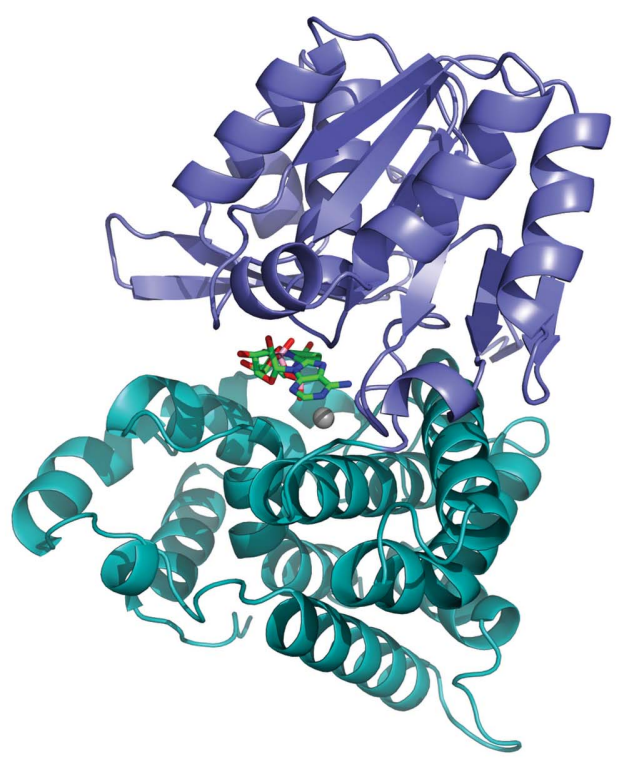

(a)

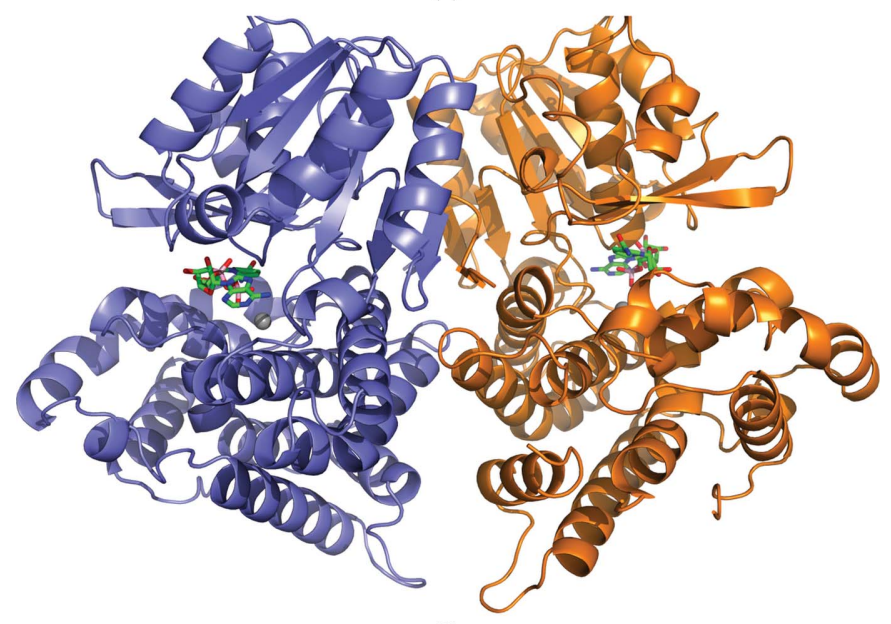

(b)

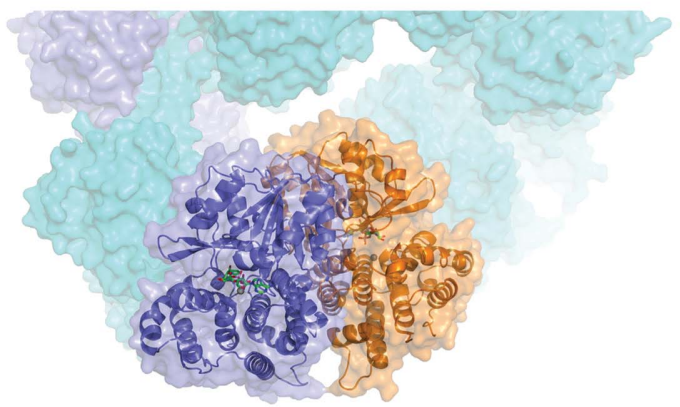

(c)

Figure 2

(a) Cartoon representation of a monomer of the ADH domain of AdhE from E. coli. The two subdomains are coloured slate (N-terminal) and teal (C-terminal), with NAD represented by green sticks and $\mathrm{Fe}^{2+}$ ions by grey spheres. (b) Cartoon representation of the oligomeric assembly, in which the two subunits forming the dimer are coloured separately. (c) Crystal structure of ADH superposed on the full-length spirosome of AdhE (PDB entry 6ahc; Kim et al., 2019). 
subdomains consists of a tight network of hydrogen bonds, as well as a salt bridge between Arg463 and Glu701. The interface area is made up of $1300 \AA^{2}$, corresponding to around $13 \%$ of the overall surface-accessible surface area of the ADH subunit.

The electron-density maps of $\mathrm{ADH}$ crystallized in the tetragonal space group showed extraneous features in the region of the conserved NAD-binding site, which is situated between the two subdomains of ADH (Fig. 2a). Consequently, NAD was modelled into these features and refined to an occupancy of 0.6. Polder maps were calculated (Liebschner et $a l ., 2017$ ) to confirm the positioning of the ligand (Fig. 3a).

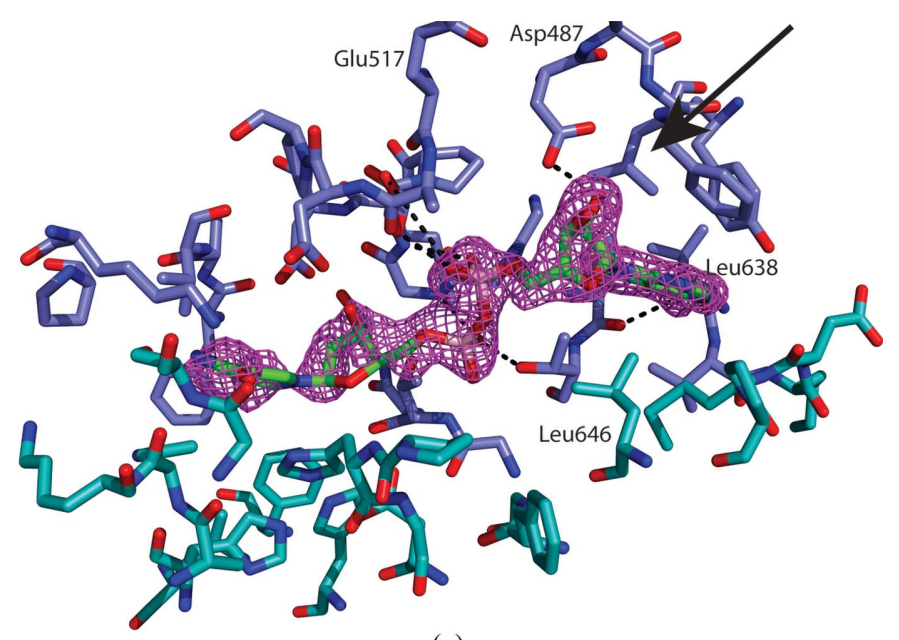

(a)

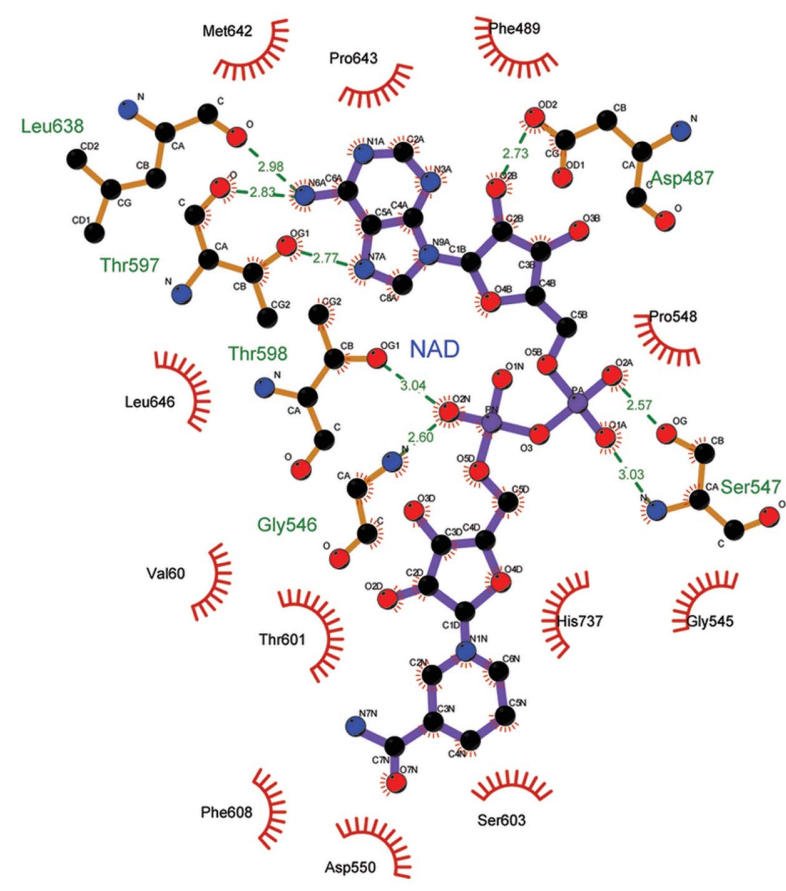

(b)

Figure 3

Close-up of the pocket where NAD is bound to the ADH structure, with a similar colour scheme as in Fig. 1(a). (a) Calculated polder maps at $3 \sigma$, showing the electron density for the ligand at an occupancy of 0.6 ; the arrow highlights where a clash would occur if NAD were substituted by NADP. (b) LIGPLOT figure of NAD and interactions with the protein environment.
NAD sits in the cleft formed between the two subdomains comprising $\mathrm{ADH}$ and the binding is mostly hydrophobic, with hydrogen bonds formed between Asp487, Gly546, Thr597 and Leu638 and the adenosine part of the NAD moiety and with Ser547 coordinating the phosphates (Fig. 3b). The benzamide part of the NAD molecule is less ordered in the electron density, and it is likely that this part does not form strong interactions with $\mathrm{ADH}$, allowing the moiety to exhibit a number of conformations. This is reflected in the $B$ factors of the NAD moiety, where the adenosine diphosphate has an average $B$ factor of $26 \AA^{2}$ and the ribose-benzamide end has an average $B$ factor of $49 \AA^{2}$, which is higher than the average $B$ factor of the protein model of $28 \AA^{2}$. NAD interactions correspond to $3 \%$ of the total accessible surface area of $\mathrm{ADH}$. The conserved residue Asp487 has been demonstrated to be important for the preference for NAD over NADP in alcohol dehydrogenases (Zheng et al., 2015). When looking at the structure (Fig. $3 a$ ) it becomes apparent that the presence of the Asp side chain will cause a steric clash with the additional phosphate group present in NADP, whereas the previously reported Asp-to-Gly mutation (Zheng et al., 2015) will allow the binding of both NAD and NADP.

Additional electron density was observed in the metal ionbinding site, as previously found in $\mathrm{gADH}$, where it was identified as $\mathrm{Zn}^{2+}$. As $\mathrm{ADH}$ has been described as being reliant on binding to iron (Holland-Staley et al., 2000) this density has been modelled as $\mathrm{Fe}^{2+}$, although it may also be a $\mathrm{Zn}^{2+}$ ion as observed in the homologue from G. thermoglucosidasius. The metal ion is coordinated by Asp653, His657, His723 and His737 with additional waters (Fig. 4).

\subsection{Oligomeric state}

As has been shown previously, the alcohol dehydrogenase domain of AdhE forms homodimers that are essential for the formation of the larger full-length AdhE spirosomes. As in $\mathrm{gADH}, \mathrm{ADH}$ crystallized as a dimer in the asymmetric unit, and the contacts between the two subunits comprise approximately $1550 \AA^{2}$, which is $10 \%$ of the accessible surface area of each subunit. This is similar to the buried surface area in the interface between the two subdomains that make up an ADH monomer.

To confirm the dimerization of ADH, small-angle X-ray scattering experiments were undertaken. A linear Guinier

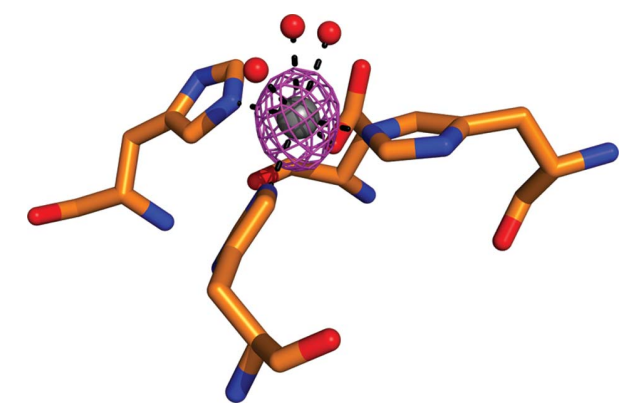

Figure 4

$\mathrm{Fe}^{2+}$ ion modelled in the electron density in the conserved metal ion site in the calculated polder map at $12 \sigma$. 
Table 5

PDB files used for structural alignment.

\begin{tabular}{|c|c|c|c|c|c|}
\hline PDB code & Protein & Species & R.m.s.d. $(\AA)$ & No. of $\mathrm{C}^{\alpha}$ atoms & Reference \\
\hline $3 z d r$ & Alcohol dehydrogenase domain & G. thermoglucosidasius & 1.2 & 373 & Extance et al. (2013) \\
\hline $4 \mathrm{fr} 2$ & Alcohol dehydrogenase & Oenococcus oeni & 1.2 & 352 & Elleuche et al. (2013) \\
\hline $2 \mathrm{bl} 4$ & Lactaldehyde oxidoreductase & E. coli & 1.3 & 356 & Montella et al. (2005) \\
\hline $1 \mathrm{rrm}$ & Lactaldehyde oxidoreductase & E. coli & 1.4 & 348 & $\begin{array}{l}\text { New York SGX Research Center for } \\
\text { Structural Genomics (unpublished work) }\end{array}$ \\
\hline
\end{tabular}

region gave a radius of gyration $\left(R_{\mathrm{g}}\right)$ of $32.5 \AA$, whereas the $R_{\mathrm{g}}$ calculated for the crystal structure is $28.1 \AA$. The discrepancy between the two values may be caused by the residues that are not accounted for by the electron density (the loop of residues 755-769 and the C-terminal residues 869-891). This difference in $R_{\mathrm{g}}$ values was not taken into consideration when calculating the $R_{\mathrm{g}}$ of the crystal structure, which could leave this value lower than it should be. Alternatively, these stretches of residues may be flexible or disordered in solution, which will

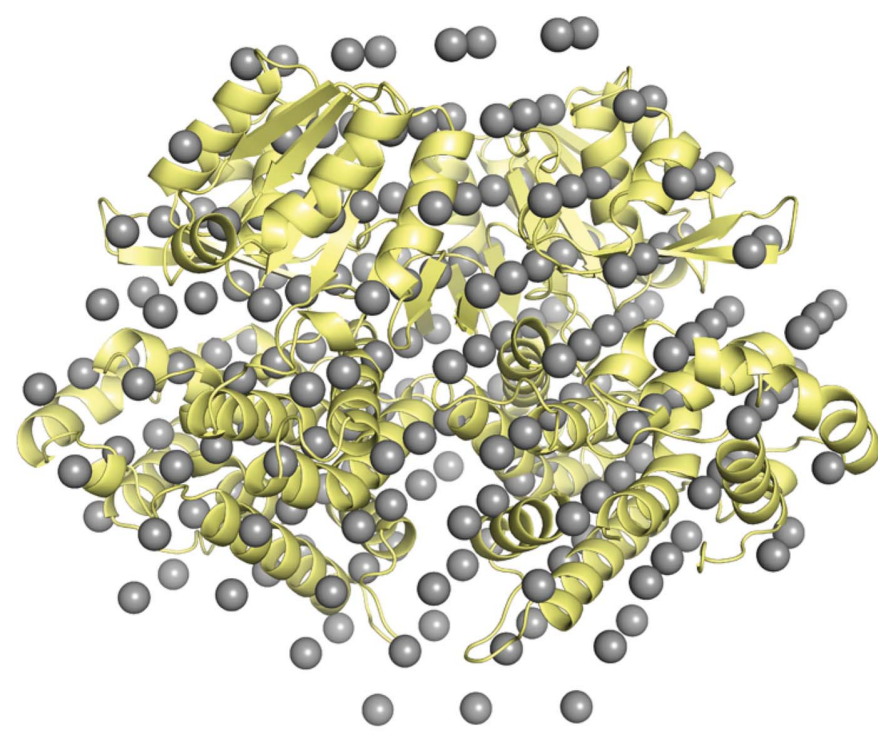

(a)

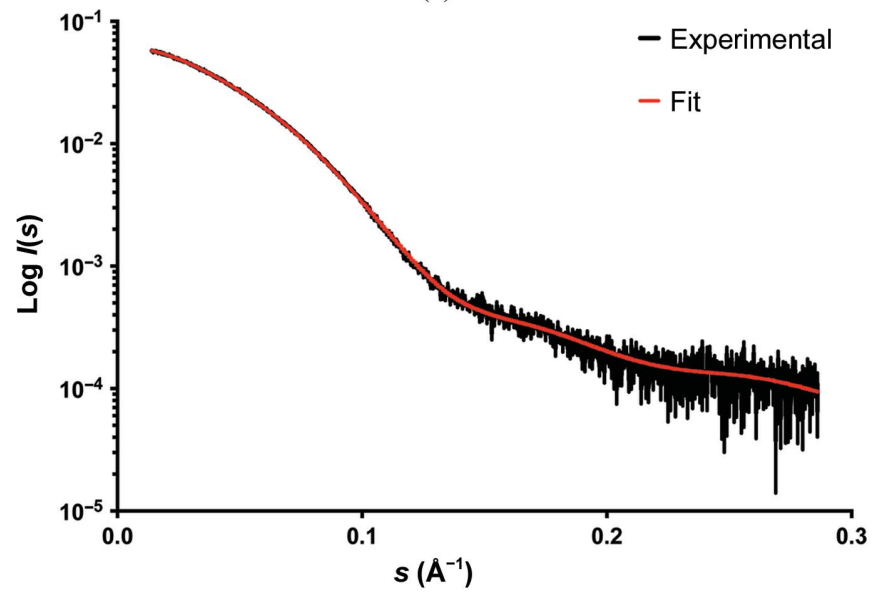

(b)

Figure 5

(a) Crystal structure of the apo form of ADH superposed on the ab initio surface envelope determined by SAXS. (b) Experimental data and fit of the $a b$ initio surface envelope (SASDB ID SASDC72). add additional scattering and will be interpreted as a larger $R_{\mathrm{g}}$. The pairwise distance distribution function $p(r)$ was calculated with GNOM (Svergun, 1992), using a $D_{\max }$ of $161 \AA$. Ab initio models were calculated using DAMMIF (Franke \& Svergun, 2009). The crystal structure was superposed on the averaged and filtered model with a good fit (Fig. 5). Rigid-body fitting of the crystal structure against the experimental SAXS data using FoXS (Schneidman-Duhovny et al., 2010) also gave a good fit, with a $\chi^{2}$ of 2.54 , again demonstrating that the crystal structure is a good representation of ADH in solution. The DAMMIF model and SAXS data for ADH have been deposited in SASBDB (Valentini et al., 2015) as entry SASDC72.

\section{Discussion}

Structures of the alcohol dehydrogenase domain from the bifunctional alcolhol/aldehyde dehydrogenase AdhE are reported here in the apo form and bound to the cofactor NAD at high resolutions. When searching for similar structures using the protein structure comparison service PDBeFold at EBI (Krissinel \& Henrick, 2004), we found six structures (using a $Q$-value of 0.7 as a cutoff) determined by X-ray crystallography, which are all prokaryotic dehydrogenases with metal ions and NAD as cofactors (Table 5). When superposed over the $\mathrm{C} \alpha$ backbone, they all superpose with reasonable r.m.s.d. values of around $1.2 \AA$, suggesting that the subunit of ADH is structurally highly conserved, whereas the sequence identity between $\mathrm{ADH}$ and the individual dehydrogenases is between $30 \%$ and $35 \%$. The structure that varies most is a lactaldehyde dehydrogenase from E. coli (PDB entry 1rrm; New York SGX Research Center for Structural Genomics, unpublished work), with an r.m.s.d. of $1.4 \AA$; the sequence identity between this protein and $\mathrm{ADH}$ is $31 \%$. When using RAPIDO, a web server that superposes a number of protein structures (Mosca \& Schneider, 2008) and identifies domains or part of domains that do not move versus those that do move, it is clear that the differences between the structures are located around a single helix in the $\mathrm{N}$-terminal subdomain and a helix-turn-helix in the C-terminal domain (Fig. 6). It is clear that the structural conservation of this domain is high throughout.

Recently, a high-resolution structure of full-length AdhE was determined by the Song group (Kim et al., 2019) using cryoEM, where they described that residue Phe670 (using E. coli $\mathrm{K}-12$ numbering) is crucial for maintenance of the spirosome structure. Superposition of the ADH crystal 


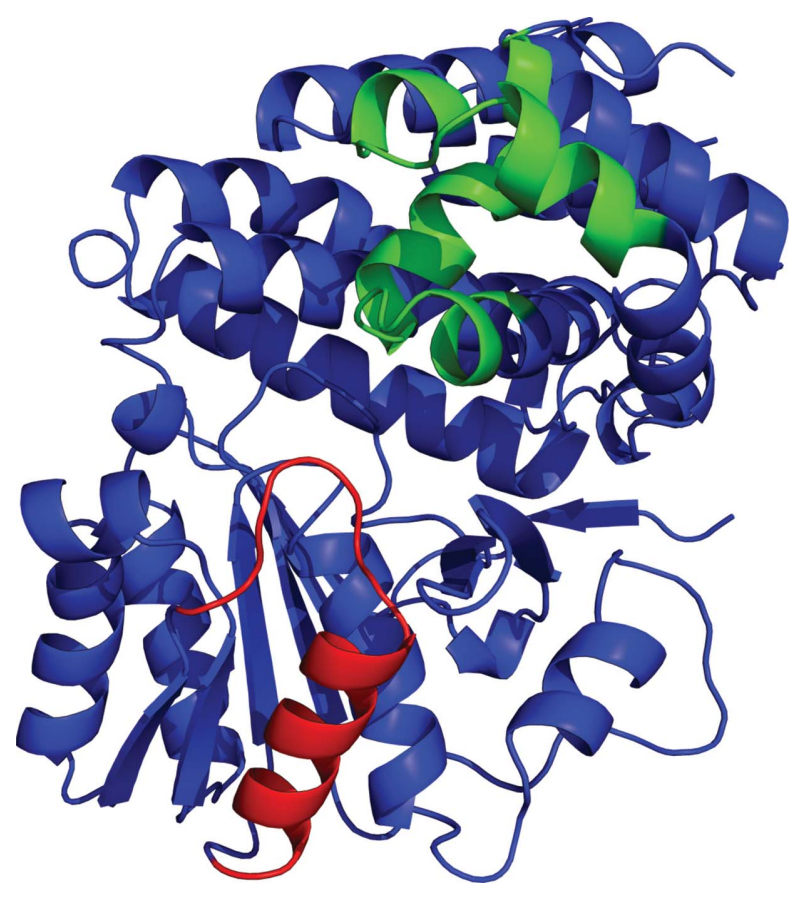

Figure 6

Structure of an $\mathrm{ADH}$ monomer, highlighting the regions that superpose less well when using RAPIDO. The $\mathrm{N}$-terminal section with higher flexibility is in red and the C-terminal section with higher flexibility is in green.

structure with the ADH domain from the full-length AdhE structure gave an r.m.s.d. of $1.21 \AA$, indicating conservation of the dimeric ADH in the spirosomes. Residues 755-769 were unaccounted for in the ADH electron-density maps. However, in the full-length cryoEM structure they are present and interact with the AldDH domain of AdhE. These residues are also missing in the structure of gADH, but this loop does not exist in the other structures, which are all monofunctional. It appears that these residues are stabilized by the presence of AldDH and play a role in coordinating the two domains in relation to each other.

Kim et al. (2019) hypothesized that hydrophobic interactions surrounding Phe670 are crucial for the complete AdhE spirosome structure. Upon the substitution of Phe670 by Glu, the spirosome structure was disrupted (Kim et al., 2019). Using PISA (Krissinel \& Henrick, 2007) to calculate the interfaces involved in the formation of the ADH dimer, we observe that the number of atoms involved in the dimer interface is reduced by around 100 in the Phe670 mutant. Disruption of the hydrophobic interaction through the mutation of Phe670 to glutamic acid was found to break the spirosome assembly into mixtures of dimeric AdhE and other higher oligomeric spirosomes. The SAXS model of the mutant Glu670-AdhE (SASBDB ID SASDGN2; Kim et al., 2019) shows the fulllength AdhE dimers to be connected through the AldDH molecules rather than the ADH domains. This finding indicates the importance of the hydrophobic interactions of Phe670 in maintaining the helical structure of the spirosome and possibly the dimer conformation of ADH.
Finally, our data here present the high-resolution crystal structures of both apo and NAD-bound forms of the alcohol dehydrogenase domain of AdhE from E. coli O157:H7 and confirm the oligomeric state and solution structure using SAXS. We also show that the ADH fold is conserved even though there is low sequence identity, and that an inserted loop in the C-terminal part of ADH appears to be involved in coordination of the two domains of the bifunctional AdhE. With the availability of the crystal structure of $\mathrm{ADH}$, future work could explore the mechanism of action of antivirulence compounds. Since AdhE has been shown to be important both as a tool for biofuel production as well as in virulence regulation (Beckham et al., 2014), a complete mechanistic understanding would provide a better understanding of the mechanism of action of the protein and how it relates to both bacterial virulence and ethanol production.

\section{Acknowledgements}

We thank Diamond Light Source for the MX and SAXS beamtime (MX12112 and MX11651, respectively) and the BL21 beamline scientists for excellent scientific support.

\section{Funding information}

This work is partially supported by the Skim Latihan Bumiputera from the Ministry of Higher Education Malaysia and Universiti Sains Islam Malaysia.

\section{References}

Beckham, K. S. H., Connolly, J. P. R., Ritchie, J. M., Wang, D., Gawthorne, J. A., Tahoun, A., Gally, D. L., Burgess, K., Burchmore, R. J., Smith, B. O., Beatson, S. A., Byron, O., Wolfe, A. J., Douce, G. R. \& Roe, A. J. (2014). Mol. Microbiol. 93, 199-211.

Elleuche, S., Fodor, K., Klippel, B., von der Heyde, A., Wilmanns, M. \& Antranikian, G. (2013). Appl. Microbiol. Biotechnol. 97, 89638975.

Emsley, P., Lohkamp, B., Scott, W. G. \& Cowtan, K. (2010). Acta Cryst. D66, 486-501.

Extance, J., Crennell, S. J., Eley, K., Cripps, R., Hough, D. W. \& Danson, M. J. (2013). Acta Cryst. D69, 2104-2115.

Franke, D., Petoukhov, M. V., Konarev, P. V., Panjkovich, A., Tuukkanen, A., Mertens, H. D. T., Kikhney, A. G., Hajizadeh, N. R., Franklin, J. M., Jeffries, C. M. \& Svergun, D. I. (2017). J. Appl. Cryst. 50, 1212-1225.

Franke, D. \& Svergun, D. I. (2009). J. Appl. Cryst. 42, 342-346.

Guinier, A. (1939). Ann. Phys. 11, 161-237.

Hills, C. (2015). PhD thesis. University of Bath, United Kingdom.

Holland-Staley, C. A., Lee, K., Clark, D. P. \& Cunningham, P. R. (2000). J. Bacteriol. 182, 6049-6054.

Kawata, T., Masuda, K. \& Ueki, Y. (1976). J. Electron Microsc. (Tokyo), 25, 283-288.

Kim, G., Azmi, L., Jang, S., Jung, T., Hebert, H., Roe, A. J., Byron, O. \& Song, J.-J. (2019). Nat. Commun. 10, 4527.

Kleywegt, G. J. \& Jones, T. A. (1994). Proceedings of the CCP4 Study Weekend. From First Map to Final Model, edited by S. Bailey, R. Hubbard \& D. Waller, pp. 59-66. Warrington: Daresbury Laboratory.

Kofoid, E., Rappleye, C., Stojiljkovic, I. \& Roth, J. (1999). J. Bacteriol. 181, 5317-5329.

Krissinel, E. \& Henrick, K. (2004). Acta Cryst. D60, 2256-2268.

Krissinel, E. \& Henrick, K. (2007). J. Mol. Biol. 372, 774-797. 
Laurenceau, R., Krasteva, P. V., Diallo, A., Ouarti, S., Duchateau, M., Malosse, C., Chamot-Rooke, J. \& Fronzes, R. (2015). PLoS Pathog. 11, e1004835.

Létoffé, S., Chalabaev, S., Dugay, J., Stressmann, F., Audrain, B., Portais, J.-C., Letisse, F. \& Ghigo, J.-M. (2017). PLoS Genet. 13, e1006800.

Liebschner, D., Afonine, P. V., Baker, M. L., Bunkóczi, G., Chen, V. B., Croll, T. I., Hintze, B., Hung, L.-W., Jain, S., McCoy, A. J., Moriarty, N. W., Oeffner, R. D., Poon, B. K., Prisant, M. G., Read, R. J., Richardson, J. S., Richardson, D. C., Sammito, M. D., Sobolev, O. V., Stockwell, D. H., Terwilliger, T. C., Urzhumtsev, A. G., Videau, L. L., Williams, C. J. \& Adams, P. D. (2019). Acta Cryst. D75, 861-877.

Liebschner, D., Afonine, P. V., Moriarty, N. W., Poon, B. K., Sobolev, O. V., Terwilliger, T. C. \& Adams, P. D. (2017). Acta Cryst. D73, $148-157$.

Lo, J., Zheng, T., Hon, S., Olson, D. G. \& Lynd, L. R. (2015). J. Bacteriol. 197, 1386-1393.

Marcal, D., Rego, A. T., Carrondo, M. A. \& Enguita, F. J. (2009). J. Bacteriol. 191, 1143-1151.

McCoy, A. J., Grosse-Kunstleve, R. W., Adams, P. D., Winn, M. D., Storoni, L. C. \& Read, R. J. (2007). J. Appl. Cryst. 40, 658-674.

Montella, C., Bellsolell, L., Pérez-Luque, R., Badía, J., Baldoma, L., Coll, M. \& Aguilar, J. (2005). J. Bacteriol. 187, 4957-4966.
Moon, J.-H., Lee, H.-J., Park, S. Y., Song, J. M., Park, M.-Y., Park, H.-M., Sun, J., Park, J.-H., Kim, B. Y. \& Kim, J.-S. (2011). J. Mol. Biol. 407, 413-424.

Mosca, R. \& Schneider, T. R. (2008). Nucleic Acids Res. 36, W42-W46. Müller, M., Mentel, M., van Hellemond, J. J., Henze, K., Woehle, C., Gould, S. B., Yu, R.-Y., van der Giezen, M., Tielens, A. G. M. \& Martin, W. F. (2012). Microbiol. Mol. Biol. Rev. 76, 444-495.

Schneidman-Duhovny, D., Hammel, M. \& Sali, A. (2010). Nucleic Acids Res. 38, W540-W544.

Svergun, D. I. (1992). J. Appl. Cryst. 25, 495-503.

Tian, L., Cervenka, N. D., Low, A. M., Olson, D. G. \& Lynd, L. R. (2019). Sci. Rep. 9, 1736.

Ueki, Y., Masuda, K. \& Kawata, T. (1982). Microbiol. Immunol. 26, 199-211.

Valentini, E., Kikhney, A. G., Previtali, G., Jeffries, C. M. \& Svergun, D. I. (2015). Nucleic Acids Res. 43, D357-D363.

Winn, M. D., Ballard, C. C., Cowtan, K. D., Dodson, E. J., Emsley, P., Evans, P. R., Keegan, R. M., Krissinel, E. B., Leslie, A. G. W., McCoy, A., McNicholas, S. J., Murshudov, G. N., Pannu, N. S., Potterton, E. A., Powell, H. R., Read, R. J., Vagin, A. \& Wilson, K. S. (2011). Acta Cryst. D67, 235-242.

Zheng, T., Olson, D. G., Tian, L., Bomble, Y. J., Himmel, M. E., Lo, J., Hon, S., Shaw, A. J., van Dijken, J. P. \& Lynd, L. R. (2015). J. Bacteriol. 197, 2610-2619. 